\title{
Effectiveness and Safety of Apixaban versus Warfarin as Outpatient Treatment of Venous Thromboembolism in U.S. Clinical Practice
}

\author{
Derek Weycker ${ }^{1}$ Xiaoyan Li ${ }^{2}$ Gail DeVecchis Wygant ${ }^{2}$ Theodore Lee ${ }^{3}$ Melissa Hamilton ${ }^{2}$ \\ Xuemei Luo ${ }^{4}$ Lien Vo ${ }^{2}$ Jack Mardekian ${ }^{3}$ Xianying Pan ${ }^{5} \quad$ Leah Burns $^{2} \quad$ Mark Atwood $^{1}$ \\ Ahuva Hanau ${ }^{1}$ Alexander T. Cohen ${ }^{6}$
}

1 Policy Analysis Inc. (PAI), Brookline, Massachusetts, United States
2 Bristol-Myers Squibb, Lawrenceville, New Jersey, United States
3 Pfizer Inc., New York, New York, United States
${ }^{4}$ Pfizer Inc., Groton, Connecticut, United States
5 Bristol-Myers Squibb, Wallingford, Connecticut, United States
6 Guy's and St. Thomas' Hospitals, London, United Kingdom

Thromb Haemost 2018;118:1951-1961.

\begin{abstract}
Address for correspondence Derek Weycker, PhD, Policy Analysis Inc. (PAI), Four Davis Court, Brookline, Massachusetts, 02445, United States (e-mail: dweycker@pai2.com).
\end{abstract}

\begin{abstract}
Keywords

- apixaban

- warfarin

- venous thromboembolism

- bleeding

In the AMPLIFY clinical trial, apixaban was non-inferior to warfarin plus subcutaneous enoxaparin bridge therapy in the treatment of acute venous thromboembolism (VTE) and was associated with significantly less bleeding. This study evaluated their comparative effectiveness and safety in routine clinical practice. A matched-cohort design and data from four U.S. private health care claims databases were employed. Study population comprised patients who initiated outpatient treatment with apixaban versus warfarin (plus parenteral anticoagulant bridge therapy) within 30 days of their initial VTE episode; apixaban and warfarin patients were matched on age, characteristics of VTE episode, study database and propensity score. Major bleeding, clinically relevant non-major (CRNM) bleeding and recurrent VTE during the 180-day (maximum) follow-up period were compared using shared frailty models. During mean follow-up of 143 days among apixaban patients $(n=17,878)$ and 152 days among warfarin patients $(n=17,878)$, incidence proportions for apixaban versus warfarin, respectively, were $1.7 \%$ versus $2.3 \%$ for major bleeding, $7.0 \%$ versus $9.4 \%$ for CRNM bleeding and $2.3 \%$ versus $2.9 \%$ for recurrent VTE. In shared frailty models, risks of major bleeding (hazard ratio $[\mathrm{HR}]=0.75$, $95 \%$ confidence interval $[\mathrm{Cl}]=0.64-0.87)$, CRNM bleeding $(\mathrm{HR}=0.77,95 \% \mathrm{Cl}=0.71-$ $0.83)$ and recurrent $\mathrm{VTE}(\mathrm{HR}=0.80,95 \% \mathrm{Cl}=0.70-0.91)$ were lower for apixaban versus warfarin. In this large-scale evaluation of VTE patients receiving outpatient treatment with apixaban or warfarin in U.S. clinical practice, risks of major bleeding, CRNM bleeding and recurrent VTE were significantly lower among patients who received apixaban.
\end{abstract}

\section{Introduction}

Lower extremity deep vein thrombosis (DVT) and pulmonary embolism (PE)-collectively referred to as venous thromboembolism (VTE)-are common conditions in clinical practice (annual incidence: 1-2 per 1,000 U.S. persons), are important causes of disability and death and are associated with substantial economic costs, especially among hospitalized patients. ${ }^{1-4}$ Risk factors for VTE include major surgery, major trauma, spinal cord injury, previous VTE, increasing age, received

June 4, 2018

accepted after revision August 29, 2018
DOI https://doi.org/

10.1055/s-0038-1673689. ISSN 0340-6245. (c) 2018 Georg Thieme Verlag KG Stuttgart · New York
License terms

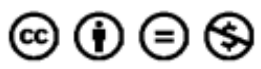


obesity, malignancy, cardiac/respiratory failure, prolonged immobility, presence of central venous lines, oestrogens and inherited/acquired haematological conditions. ${ }^{5-8}$ Around $25-50 \%$ of clinically recognized VTE cases occur in those who are neither hospitalized nor recovering from a major illness. ${ }^{5,9}$

Oral anticoagulant (OAC) therapy with vitamin K antagonists-most notably, warfarin-has long been the standard of care for VTE, but their use is associated with elevated risks of major bleeding and clinically relevant non-major (CRNM) bleeding. ${ }^{10}$ Recently, direct-acting (non-vitamin K antagonist) OACs (DOACs) - such as apixaban, dabigatran, edoxaban and rivaroxaban-have been approved globally as alternatives to warfarin. ${ }^{11-14}$ These drugs have been demonstrated in clinical trials to be non-inferior to warfarin in reducing the risk of recurrent VTE and VTE-related death, and to confer a comparable or reduced risk of bleeding. ${ }^{15-19}$ Unlike warfarin, DOACs do not require routine monitoring of the international normalized ratio (INR) and have fewer drug/food interactions. ${ }^{20}$ Moreover, since 2012, the CHEST Guidelines for Antithrombotic Therapy in VTE have specified that for the treatment of VTE in patients without cancer, DOACs are recommended over vitamin $\mathrm{K}$ antagonists. ${ }^{10,21}$

Evidence of the efficacy and safety of drugs from randomized controlled clinical trials may not necessarily be reflective of their effectiveness and safety in clinical practice, in which patient characteristics and 'real-world' treatment practices may vary substantially from those in the trial setting. In addition, use of warfarin for the treatment of VTE and prevention of recurrent VTE in patients without cancer remains common-place in current U.S. clinical practice, and while its effectiveness and safety in real-world settings have been evaluated relative to some of the currently available DOACs (e.g. rivaroxaban), they have not been evaluated relative to all of the currently available DOACs, especially those approved in more recent years (e.g. apixaban, edoxaban). ${ }^{22-28}$

Because of the limitations of published research, this new observational study was undertaken to provide real-world evidence on the comparative effectiveness and safety of apixaban versus warfarin (with parenteral anticoagulant [PAC] bridging therapy) in the treatment of patients with VTE. To increase the power of the study to evaluate outcomes of interest, and to enhance the generalizability of study findings, four U.S. private health care claims databases-including data from unique and diverse populations-were employed in analyses. While lacking certain elements of clinical detail, such databases provide access to the health profile and health care experience (across the continuum of care settings) of tens of millions of persons over a multi-year period of time, and thus contain information on large numbers of VTE patients receiving outpatient anticoagulant therapy.

\section{Materials and Methods}

\section{Study Design and Data Source}

This study employed a matched-cohort design and data from four large integrated U.S. private health care claims databases -the Truven Health Analytics' MarketScan Commercial Claims and Encounters and Medicare Supplemental and Coordination of Benefits Databases (the 'MarketScan Database'); the IQVIA PharMetrics Plus Database (the 'PharMetrics Database'); the Optum Clinformatics Claims Database (the 'Optum Database'); and the Humana Medical, Laboratory, and Pharmacy Claims Database (the 'Humana Database') (ClinicalTrials.gov Identifier: NCT03521908). Patient-level data from the four databases spanned from 1 March 2014 through 30 June 2017 and were pooled for analyses. A detailed description of the study design, data sources and study methods may be found in - Supplementary Material A (available in the online version), and operational algorithms/codes employed to define study variables may be found in - Supplementary Material B (available in the online version).

\section{Source and Study Populations}

The source population included all patients aged $\geq 18$ years who, between 1 September 2014 and 30 June 2017, had an encounter for the treatment of VTE. The window for identification of study subjects spans the period from the U.S. Food and Drug Administration approval date for use of apixaban in the treatment of VTE through the end of the study databases. Evidence of VTE was ascertained based on encounters in the acute-care inpatient setting or outpatient setting (e.g. emergency department, physician office) with International Classification of Diseases, Ninth/Tenth Revision, Clinical Modification (ICD-9-CM/ICD-10-CM) diagnosis codes for lower extremity DVT or PE in any position. The earliest such encounter for each patient was designated the 'index encounter'. Outpatient encounters followed by inpatient encounters within 7 days were considered as inpatient index encounters, unless apixaban or warfarin was initiated between the outpatient encounter and inpatient encounter, in which case they were classified as outpatient index encounters.

From the source population, all patients who received outpatient treatment with apixaban or warfarin (plus PAC bridging therapy) following their index encounters, and who met all other inclusion criteria, were selected as candidates for matching and thus inclusion in the study population. Outpatient use of apixaban or warfarin from the date of the index encounter (service date, if outpatient VTE; discharge date, if inpatient VTE) through the 30-day period thereafter was ascertained based on the National Drug Codes (NDCs). The first treatment (i.e. apixaban or warfarin) received by each patient was designated as the 'index therapy'. Warfarin patients with an index VTE encounter requiring outpatient care only who did not have evidence of PAC use (i.e. bridging therapy) during the period \pm 14 days from first receipt of warfarin, and those who had evidence of PAC use during this period and received it beyond the 14-day period following initiation of warfarin, were excluded. Other inclusion/exclusion criteria (e.g. $\geq 6$ months of enrolment in a participating health plan prior to index encounter; no evidence of atrial fibrillation/flutter, prior VTE, history of bleeding, active malignancy) are listed in -Supplementary Material A (available in the online version).

The matching of apixaban patients to warfarin patients was implemented in two steps. First, for each apixaban patient (sorted randomly, and beginning with the first patient), all warfarin patients with exact matches on age 
(in years), study database (MarketScan, PharMetrics, Optum or Humana) and each characteristic of the qualifying VTE event-including care setting (inpatient vs. ambulatory), VTE diagnosis (DVT only vs. PE [with or without DVT]), VTE aetiology (provoked vs. unprovoked)-were selected as candidates for matching. Second, from the pool of candidates, the warfarin patient with the closest estimated propensity score was matched 1:1, without replacement, using the nearest-neighbour approach. Provoked VTE was defined as an event that was preceded (within 3 months) by hormone therapy, fracture/trauma involving lower extremities, pelvic/ orthopaedic surgery or hospitalization for medical or surgical reasons. Propensity scores for receipt of apixaban versus warfarin-the dependent variable-were estimated using multivariable logistic regression; independent variables included age, gender, comorbidity profile, history of fall(s), history of fracture/trauma involving lower extremities, history of selected surgeries and outpatient pharmacotherapy (see - Supplementary Material A, Other Study Variables section, for a complete list of independent variables used in the estimation of propensity scores, available in the online version).

\section{Study Outcomes}

Study outcomes included major bleeding, CRNM bleeding and recurrent VTE. Outcomes were ascertained from the day after initiation of index therapy through the subsequent 180 day period, date of health plan disenrollment, date of death (in hospital), date of index therapy discontinuation, date of switch to another OAC, date of initiation of (new) PAC treatment or end of study database (30 June 2017), whichever occurred first.

Major bleeding was defined as an acute-care inpatient admission with a principal or first-listed ICD-9-CM/ICD-10CM diagnosis code for gastrointestinal (GI) bleeding, intracranial haemorrhage or other selected types/sites of bleeding, or an ICD-9-CM/ICD-10-CM procedure code for the treatment of bleeding (see - Supplementary Material A, Endpoint(s)/Outcomes(s) Assessment section, for a complete list of diagnosis codes, available in the online version). CRNM bleeding was defined as an acute-care inpatient admission with a secondary diagnosis code or an ambulatory care encounter with a diagnosis code for GI bleeding or other non-critical care types/sites of bleeding. Events that met the definitions for both major bleeding and CRNM bleeding were classified as major bleeding; CRNM bleeding events that followed major bleeding events were not considered in analyses of CRNM bleeding. Recurrent VTE was defined as a subsequent acute-care inpatient admission with a corresponding principal/first-listed diagnosis; admissions occurring within 7 days of the index VTE encounter-irrespective of care setting-were not considered as recurrent events.

\section{Statistical Analyses}

The adequacy of the matching procedure in terms of patients' baseline characteristics was evaluated using standardized differences; a value of $<0.1$ was assumed to indicate a negligible difference in the characteristic between apixaban patients and warfarin patients. ${ }^{29,30}$ Major bleeding, CRNM bleeding and recurrent VTE were compared between patients who received apixaban versus warfarin using shared frailty models (an extension of the Cox proportional hazards model that adjusts for correlation from matching). The Kaplan-Meier method was used to generate the cumulative incidence of major bleeding, CRNM bleeding and recurrent VTE, during the follow-up period and statistical comparisons were based on the stratified log-rank test. The proportional hazards assumption was evaluated using published methods. ${ }^{31,32}$ Based on a priori assumptions regarding sample size and risks of major bleeding, CRNM bleeding and recurrent VTE, analyses were adequately powered to evaluate study objectives; a description of power calculations may be found in - Supplementary Material A, Power/Sample Size section (available in the online version). The sensitivity of study results to alternative methods for confounding adjustment (i.e. using all patients and the inverse probability treatment weighting method and multivariable Cox proportional hazards models, respectively), and the variability of study results when using data from each of the four health care claims repositories separately, were evaluated. Methods for these analyses are set forth in -Supplementary Material A (available in the online version).

\section{Results}

\section{Patient Selection and Patient Characteristics}

Approximately 1.4 million adult patients had a diagnosis of VTE between September 2014 and June 2017, of whom 285,042 (21\%) had $\geq 1$ filled prescription for apixaban ( $n=48,239)$ or warfarin $(n=236,803)$ during the 30-day period following their index VTE encounter (-Fig. 1). Among this sub-group, 17,878 (87\%) of the 20,561 apixaban patients who met all remaining selection criteria were matched (1:1) to warfarin patients, and all matched patients were included in the study population. A detailed description of patients meeting inclusion/exclusion criteria may be found in -Supplementary Material C (available in the online version). Mean (standard deviation [SD]) age of matched apixaban and warfarin patients was 60 (16) years, 37\% of both treatment groups were aged $\geq 65$ years and $52 \%$ of apixaban and warfarin patients were male; comorbidity profiles, history of procedures and use of outpatient pharmacotherapy were also comparable (-Table 1). Fifty-four per cent of apixaban and warfarin patients were hospitalized for their index VTE encounter, $41 \%$ had PE (with or without DVT) and 23\% had provoked VTE. Mean time from index VTE encounter to initial dispense of index therapy was 6.9 days for apixaban patients and 3.7 days for warfarin patients.

\section{Use of Index and Other Therapies}

During a mean (SD) follow-up of 143 (57) days, apixaban patients filled 4.2 (2.9) outpatient prescriptions covering 116 (61) therapy days (-Supplementary Material C, available in the online version); $93 \%$ of initial prescriptions and $91 \%$ of subsequent prescriptions were for the $5 \mathrm{mg}$ tablet. For warfarin patients during an average follow-up of 152 (52) 


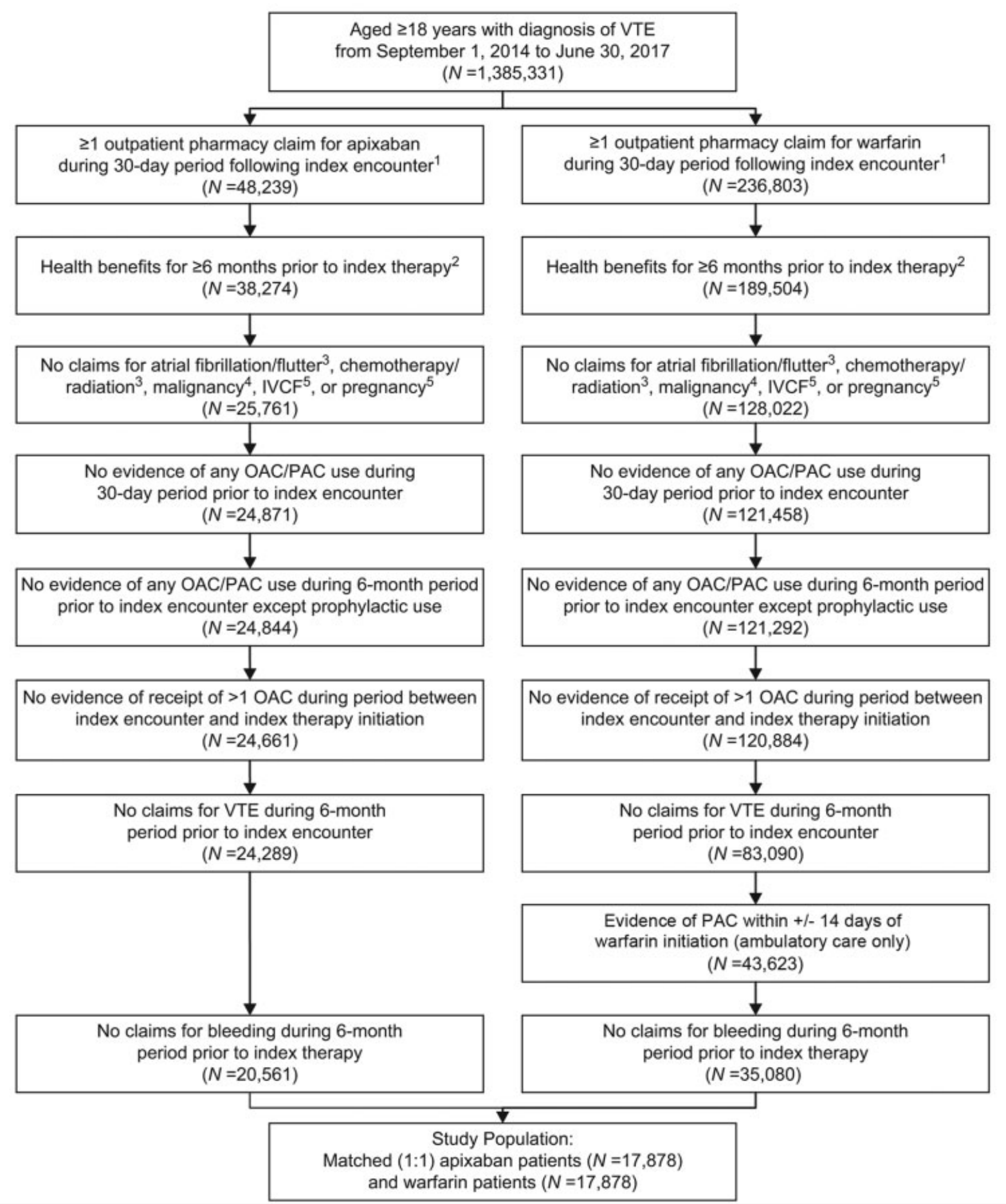

\footnotetext{
${ }^{1}$ The earliest encounter in the acute-care inpatient setting or ambulatory-care setting with ICD-9-CM/ICD-10-CM diagnosis codes for lower extremity DVT or PE for each patient was designated the "index encounter"

${ }^{2}$ First outpatient treatment with apixaban or warfarin received by each patient during the 30-day period following the index encounter was designated as the "index therapy"

${ }^{3}$ Atrial fibrillation/flutter or chemotherapy/ radiation during 6-month period prior to index therapy

${ }^{4}$ Malignancy other than non-melanoma skin cancer during 90-day period prior to index therapy

${ }^{5}$ IVCF or pregnancy during study period
}

Fig. 1 Selection of patients receiving apixaban or warfarin as outpatient therapy for VTE. IVCF, inferior vena cava filter; OAC, oral anticoagulant; PAC, parenteral anticoagulant; VTE, venous thromboembolism. 
Table 1 Characteristics of patients receiving apixaban or warfarin as outpatient therapy for VTE

\begin{tabular}{|c|c|c|c|}
\hline & \multicolumn{3}{|c|}{ Study population } \\
\hline & $\begin{array}{l}\text { Apixaban } \\
(n=17,878)\end{array}$ & $\begin{array}{l}\text { Warfarin } \\
(n=17,878)\end{array}$ & Standard difference \\
\hline \multicolumn{4}{|l|}{ Qualifying VTE encounter, $n$ (\%) } \\
\hline \multicolumn{4}{|l|}{ Diagnosis } \\
\hline PE (with or without DVT) & $7,322(41.0)$ & $7,322(41.0)$ & 0.0000 \\
\hline PE with DVT & $1,655(22.6)$ & $1,635(22.3)$ & 0.0039 \\
\hline PE without DVT & $5,667(77.4)$ & $5,687(77.7)$ & 0.0024 \\
\hline DVT only & $10,556(59.0)$ & $10,556(59.0)$ & 0.0000 \\
\hline \multicolumn{4}{|l|}{ Presumed aetiology } \\
\hline Provoked & $4,069(22.8)$ & $4,069(22.8)$ & 0.0000 \\
\hline Unprovoked & $13,809(77.2)$ & $13,809(77.2)$ & 0.0000 \\
\hline \multicolumn{4}{|l|}{ Setting } \\
\hline Acute-care inpatient & $9,683(54.2)$ & $9,683(54.2)$ & 0.0000 \\
\hline Ambulatory-care & $8,195(45.8)$ & $8,195(45.8)$ & 0.0000 \\
\hline \multicolumn{4}{|l|}{ Patient } \\
\hline \multicolumn{4}{|l|}{ Age $(y)$} \\
\hline Mean (SD) & $60.0(16.0)$ & $60.0(16.0)$ & 0.0000 \\
\hline \multicolumn{4}{|l|}{ Sex, $n(\%)$} \\
\hline Male & $9,282(51.9)$ & $9,279(51.9)$ & 0.0003 \\
\hline Female & $8,595(48.1)$ & $8,599(48.1)$ & 0.0004 \\
\hline \multicolumn{4}{|l|}{ Comorbidity profile } \\
\hline Deyo-Charlson Comorbidity Index, mean (SD) & $1.1(1.7)$ & $1.0(1.7)$ & 0.0001 \\
\hline \multicolumn{4}{|l|}{ Comorbidities, $n(\%)$} \\
\hline Acquired immunodeficiency syndrome (AIDS) & $72(0.4)$ & $64(0.4)$ & 0.0073 \\
\hline Alcohol abuse & $591(3.3)$ & $563(3.1)$ & 0.0089 \\
\hline Anaemia & $2,562(14.3)$ & $2,560(14.3)$ & 0.0003 \\
\hline Bleeding & $277(1.5)$ & $263(1.5)$ & 0.0064 \\
\hline Central venous catheter & $689(3.9)$ & $635(3.6)$ & 0.0160 \\
\hline Cerebrovascular disease (stroke/TIA) & $1,216(6.8)$ & $1,242(6.9)$ & 0.0057 \\
\hline Chronic obstructive pulmonary disease (COPD) & $2,155(12.1)$ & $2,163(12.1)$ & 0.0014 \\
\hline Coagulopathy & $222(1.2)$ & $218(1.2)$ & 0.0020 \\
\hline Congestive heart failure & $1,934(10.8)$ & $1,873(10.5)$ & 0.0111 \\
\hline Ischaemic heart/coronary artery disease & $3,464(19.4)$ & $3,247(18.2)$ & 0.0311 \\
\hline Dementia & $508(2.8)$ & $429(2.4)$ & 0.0277 \\
\hline Diabetes & $4,342(24.3)$ & $4,213(23.6)$ & 0.0169 \\
\hline Dyspepsia or stomach discomfort & $2,903(16.2)$ & $2,892(16.2)$ & 0.0017 \\
\hline Falls & $664(3.7)$ & $644(3.6)$ & 0.0060 \\
\hline Fractures involving the lower extremities & $1,695(9.5)$ & $1,812(10.1)$ & 0.0220 \\
\hline Hemiplegia or paraplegia & $187(1.0)$ & $191(1.1)$ & 0.0022 \\
\hline Hyperlipidaemia & $8,111(45.4)$ & $7,875(44.0)$ & 0.0266 \\
\hline Hypertension & $9,799(54.8)$ & $9,467(53.0)$ & 0.0373 \\
\hline Inflammatory bowel syndrome & $179(1.0)$ & $190(1.1)$ & 0.0061 \\
\hline Liver disease & $998(5.6)$ & $1,031(5.8)$ & 0.0080 \\
\hline Malignancy (excluding non-melanoma skin cancer) & $216(1.2)$ & $200(1.1)$ & 0.0083 \\
\hline
\end{tabular}


Table 1 (Continued)

\begin{tabular}{|c|c|c|c|}
\hline & \multicolumn{3}{|c|}{ Study population } \\
\hline & $\begin{array}{l}\text { Apixaban } \\
(n=17,878)\end{array}$ & $\begin{array}{l}\text { Warfarin } \\
(n=17,878)\end{array}$ & Standard difference \\
\hline Obesity & 3,551 (19.9) & 3,549 (19.9) & 0.0003 \\
\hline Peptic ulcer disease & $160(0.9)$ & $154(0.9)$ & 0.0036 \\
\hline Peripheral vascular disease & $2,037(11.4)$ & $2,052(11.5)$ & 0.0026 \\
\hline Pneumonia & $1,659(9.3)$ & $1,602(9.0)$ & 0.0111 \\
\hline Renal disease & $2,388(13.4)$ & $2,401(13.4)$ & 0.0021 \\
\hline Rheumatologic disease & $487(2.7)$ & $488(2.7)$ & 0.0003 \\
\hline Sleep apnoea & 1,955 (10.9) & $1,881(10.5)$ & 0.0134 \\
\hline Spinal cord injury & $20(0.1)$ & $12(0.1)$ & 0.0150 \\
\hline Thrombocytopaenia & $499(2.8)$ & $495(2.8)$ & 0.0014 \\
\hline Thrombophilia & $710(4.0)$ & $735(4.1)$ & 0.0071 \\
\hline Varicose veins & $741(4.1)$ & $583(3.3)$ & 0.0468 \\
\hline \multicolumn{4}{|l|}{ Procedures, $n(\%)$} \\
\hline Abdominal surgery & $2,119(11.9)$ & $2,033(11.4)$ & 0.0150 \\
\hline Haemodialysis & $131(0.7)$ & $150(0.8)$ & 0.0120 \\
\hline Hip replacement & $137(0.8)$ & $146(0.8)$ & 0.0057 \\
\hline Knee replacement & $289(1.6)$ & $322(1.8)$ & 0.0142 \\
\hline Pelvic or orthopaedic surgery & $6,732(37.7)$ & $6,693(37.4)$ & 0.0045 \\
\hline Recent surgery (major) & $1,794(10.0)$ & 1,765 (9.9) & 0.0054 \\
\hline \multicolumn{4}{|l|}{ Outpatient pharmacotherapy, $n$ (\%) } \\
\hline ACE inhibitors/ARBs & $5,175(28.9)$ & $5,032(28.1)$ & 0.0177 \\
\hline Anti-arrhythmics & $184(1.0)$ & $118(0.7)$ & 0.0403 \\
\hline Anti-platelets & $872(4.9)$ & $794(4.4)$ & 0.0207 \\
\hline Aromatase inhibitors & $80(0.4)$ & $72(0.4)$ & 0.0069 \\
\hline Beta blockers & $4,308(24.1)$ & $4,229(23.7)$ & 0.0104 \\
\hline Calcium channel blockers & $2,704(15.1)$ & $2,568(14.4)$ & 0.0215 \\
\hline Contraceptives (oral) & $627(3.5)$ & $642(3.6)$ & 0.0045 \\
\hline Erythropoiesis stimulating agents & $9(0.1)$ & $5(0.0)$ & 0.0113 \\
\hline Estrogen hormone agents & $453(2.5)$ & $388(2.2)$ & 0.0240 \\
\hline Gastroprotective agents & $3,441(19.2)$ & $3,456(19.3)$ & 0.0021 \\
\hline Non-estrogen hormone agents & $6,347(35.5)$ & $6,138(34.3)$ & 0.0245 \\
\hline NSAIDs & $3,113(17.4)$ & $3,075(17.2)$ & 0.0056 \\
\hline SERMs & $1(0.0)$ & $4(0.0)$ & 0.0142 \\
\hline Statins & $5,281(29.5)$ & $5,083(28.4)$ & 0.0244 \\
\hline
\end{tabular}

Abbreviations: ACE inhibitors, angiotensin-converting enzyme inhibitors; AIDS, acquired immune deficiency syndrome; ARBs, angiotensin receptor blockers; COPD, chronic obstructive pulmonary disease; DVT, deep vein thrombosis; NSAID, non-steroidal anti-inflammatory drug; PE, pulmonary embolism; SD, standard deviation; SERM, selective estrogen receptor modulator; TIA, transient ischaemic attack; VTE, venous thromboembolism.

days, numbers of filled outpatient prescriptions and therapy days were 5.1 (4.2) and 136 (71). Less than 5\% of patients in each treatment group switched from their index therapy to another OAC during follow-up. Outpatient use of PAC between the index encounter and initiation of index therapy was recorded among 59\% of warfarin patients and $2 \%$ of apixaban patients; PAC use subsequent to initiation of index therapy was $63 \%$ and $3 \%$, respectively.

\section{Risks of Bleeding and Recurrent VTE}

Incidence proportion for major bleeding was $1.7 \%$ (4.2 per 100 patient-years) among apixaban patients versus $2.3 \%$ (5.5 per 100 patient-years) among warfarin patients, while corresponding figures for CRNM bleeding were 7.0\% (18.0 per 100 patient-years) and 9.4\% (22.7 per 100 patient-years) (-Table 2, - Fig. 2). The hazard ratio (HR) for major bleeding was 0.75 (95\% confidence interval $[\mathrm{CI}]=0.64-0.87$ ) for 
Table 2 Risks and hazard ratios for major bleeding, CRNM bleeding and recurrent VTE among patients receiving apixaban and warfarin as outpatient therapy for VTE

\begin{tabular}{|c|c|c|c|c|c|c|c|}
\hline & No. Patients & No. Events & \% Evented & Risk per $100 \mathrm{PY}$ & HR & $95 \% \mathrm{Cl}$ & $p$-Value \\
\hline \multicolumn{8}{|l|}{ Major bleeding } \\
\hline \multicolumn{8}{|l|}{ All } \\
\hline Apixaban & 17,878 & 295 & 1.7 & 4.2 & 0.75 & $0.64-0.87$ & $<0.001$ \\
\hline Warfarin & 17,878 & 412 & 2.3 & 5.5 & - & - & - \\
\hline \multicolumn{8}{|c|}{ Gastrointestinal } \\
\hline Apixaban & 17,878 & 111 & 0.6 & 1.6 & 0.72 & $0.57-0.92$ & 0.009 \\
\hline Warfarin & 17,878 & 160 & 0.9 & 2.2 & - & - & - \\
\hline \multicolumn{8}{|l|}{ Intra-cranial } \\
\hline Apixaban & 17,878 & 24 & 0.1 & 0.3 & 0.97 & $0.56-1.69$ & 0.920 \\
\hline Warfarin & 17,878 & 26 & 0.1 & 0.3 & - & - & - \\
\hline \multicolumn{8}{|l|}{ Other } \\
\hline Apixaban & 17,878 & 160 & 0.9 & 2.3 & 0.74 & $0.60-0.90$ & 0.003 \\
\hline Warfarin & 17,878 & 226 & 1.3 & 3.0 & - & - & - \\
\hline \multicolumn{8}{|l|}{ CRNM bleeding } \\
\hline \multicolumn{8}{|l|}{ All } \\
\hline Apixaban & 17,878 & 1,257 & 7.0 & 18.0 & 0.77 & $0.71-0.83$ & $<0.001$ \\
\hline Warfarin & 17,878 & 1,688 & 9.4 & 22.7 & - & - & - \\
\hline \multicolumn{8}{|c|}{ Gastrointestinal } \\
\hline Apixaban & 17,878 & 385 & 2.2 & 5.5 & 0.88 & $0.76-1.00$ & 0.053 \\
\hline Warfarin & 17,878 & 460 & 2.6 & 6.2 & - & - & - \\
\hline \multicolumn{8}{|l|}{ Other } \\
\hline Apixaban & 17,878 & 874 & 4.9 & 12.5 & 0.73 & $0.67-0.79$ & $<0.001$ \\
\hline Warfarin & 17,878 & 1,238 & 6.9 & 16.7 & - & - & - \\
\hline \multicolumn{8}{|l|}{ Recurrent VTE } \\
\hline Apixaban & 17,878 & 403 & 2.3 & 5.8 & 0.80 & $0.70-0.91$ & $<0.001$ \\
\hline Warfarin & 17,878 & 521 & 2.9 & 7.0 & - & - & - \\
\hline
\end{tabular}

Abbreviations: $\mathrm{Cl}$, confidence interval; CRNM, clinically relevant non-major; HR, hazard ratio; PY, patient-years; VTE, venous thromboembolism.

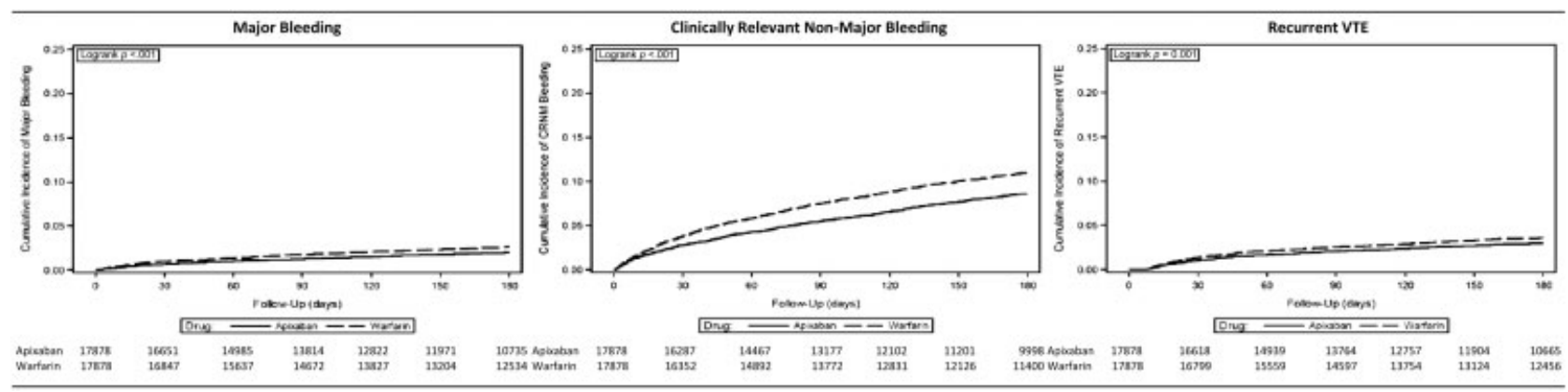

Fig. 2 Cumulative incidence of major bleeding, CRNM bleeding and recurrent VTE among patients receiving apixaban and warfarin as outpatient therapy for VTE. CRNM, clinically relevant non-major; VTE, venous thromboembolism.

apixaban versus warfarin, while the HR for CRNM bleeding was 0.77 (95\% $\mathrm{CI}=0.71-0.83)$.

Incidence proportion for recurrent VTE was $2.3 \%$ ( 5.8 per 100 patient-years) for those receiving apixaban versus $2.9 \%$ ( 7.0 per
100 patient-years) for those receiving warfarin; the HR was 0.80 $(95 \% \mathrm{CI}=0.70-0.91)$. Results from analyses based on each of the four databases separately (-Fig. 3), and those from sensitivity analyses employing alternative methods for confounding 


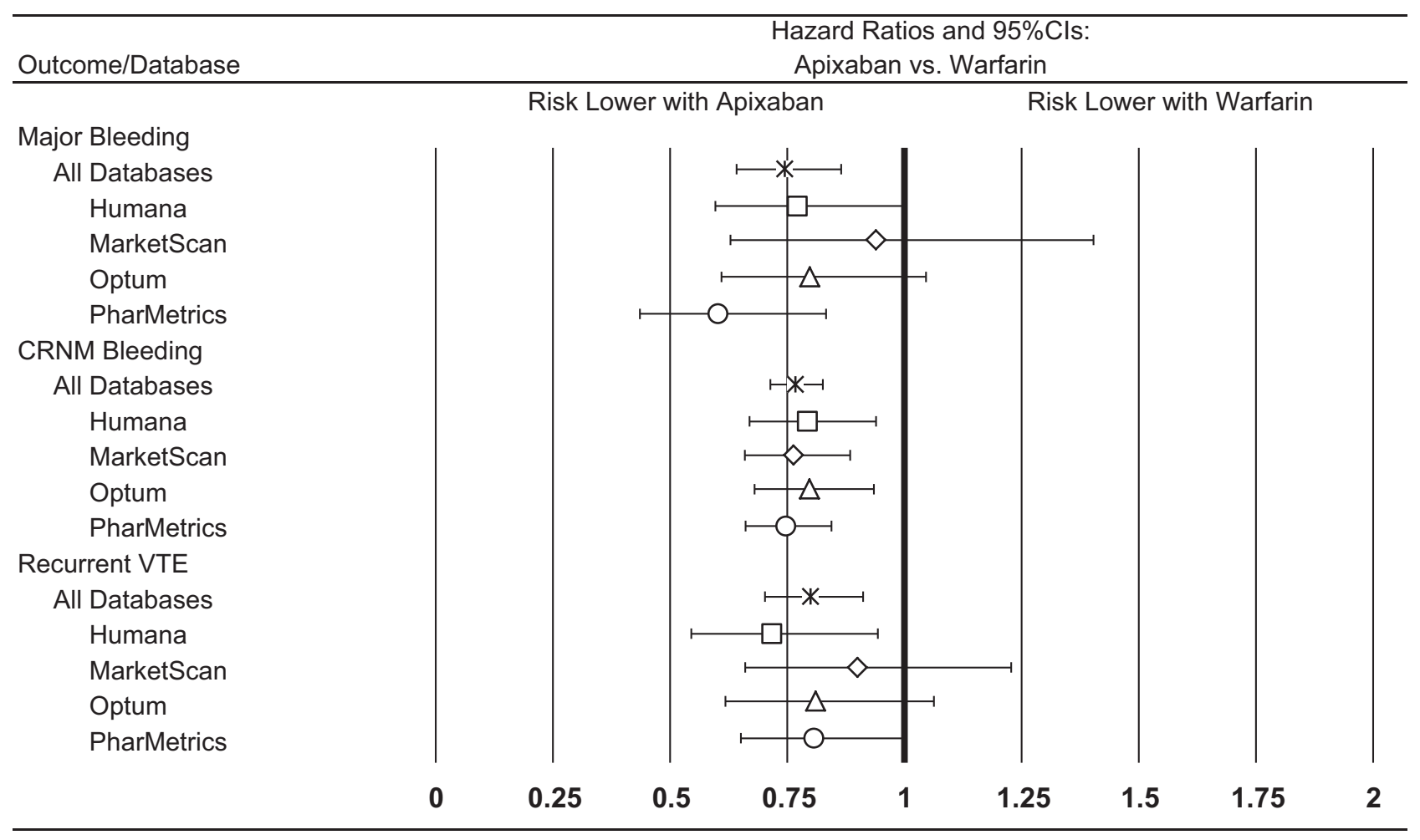

Fig. 3 Hazard ratios for bleeding events and recurrent VTE among patients receiving apixaban and warfarin as outpatient therapy for VTE, overall and by study database. Cl, confidence interval; CRNM, clinically relevant non-major; VTE, venous thromboembolism.

adjustment ( - Fig. 4, - Supplementary Material C, available in the online version), were largely similar.

\section{Discussion}

This study found that the risks of major bleeding, CRNM bleeding and recurrent VTE were significantly lower among patients receiving apixaban than among those receiving warfarin for the outpatient treatment and prevention of VTE in U.S. clinical practice. These findings are largely comparable with the efficacy and safety of apixaban versus warfarin for the treatment of VTE demonstrated in a randomized clinical trial. ${ }^{15}$ Other advantages conferred by apixaban compared with warfarin are simplified monitoring and fewer drug/food interactions. Guidelines recommend the use of DOACs (such as apixaban) over vitamin $\mathrm{K}$ antagonists (such as warfarin). However, the findings of this study suggest that outpatient use of warfarin for the treatment of VTE remains widespread in current U.S. clinical practice. $^{10,11,15,20}$

This evaluation is, to the best of our knowledge, the first one to compare the effectiveness and safety of apixaban versus warfarin for the treatment of VTE in a real-world population. Our findings are based on data for nearly 36,000 patients from four large databases-including information from unique and diverse populations-and are generally robust in sensitivity analyses when using each database separately. The conclusions that may be drawn from our findings are generally consistent with, and supplement those from, the pivotal Phase III clinical trial of apixaban versus conventional therapy (i.e. enoxaparin followed by warfarin) in the treatment of VTE (AMPLIFY). In AMPLIFY, like our study, risks of major bleeding ( $0.6 \%$ vs. $1.8 \%$; relative risk $=0.31$ [0.17-0.55], $p<0.001)$ and CRNM bleeding (3.8\% vs. $8.0 \%$; relative risk $=0.48[0.38-$ 0.60 ], $p<0.001$ ) during the 180 -day follow-up period were significantly lower with apixaban versus conventional therapy. ${ }^{15}$ Apixaban also was found to be noninferior to conventional therapy in terms of risk of recurrent VTE or VTE-related death during the 180-day followup period: $2.3 \%$ versus $2.7 \%$ (relative risk $=0.84$ [0.601.18], $p<0.001$ for non-inferiority). We note that results from our evaluation and AMPLIFY were found to be comparable despite differences in study designs, study populations and study methods, and that real-world studies are subject to the vagaries of clinical practice (e.g. treatment selection and patterns, drug compliance). For example, at baseline, our study population was slightly older (60 vs. 57 years), had a higher percentage of patients with PE (41\% vs. 34\%) and had a higher percentage of patients with provoked VTE (23\% vs. $10 \%)$. We also note that such differences may be attributable-at least in partto imprecision in the algorithms employed to characterize the population in our study.

Our study, although large with nearly 36,000 patients, is still subject to the inherent limitations of evaluations using health care claims data. In clinical practice, patients who receive apixaban may be systematically different than those 


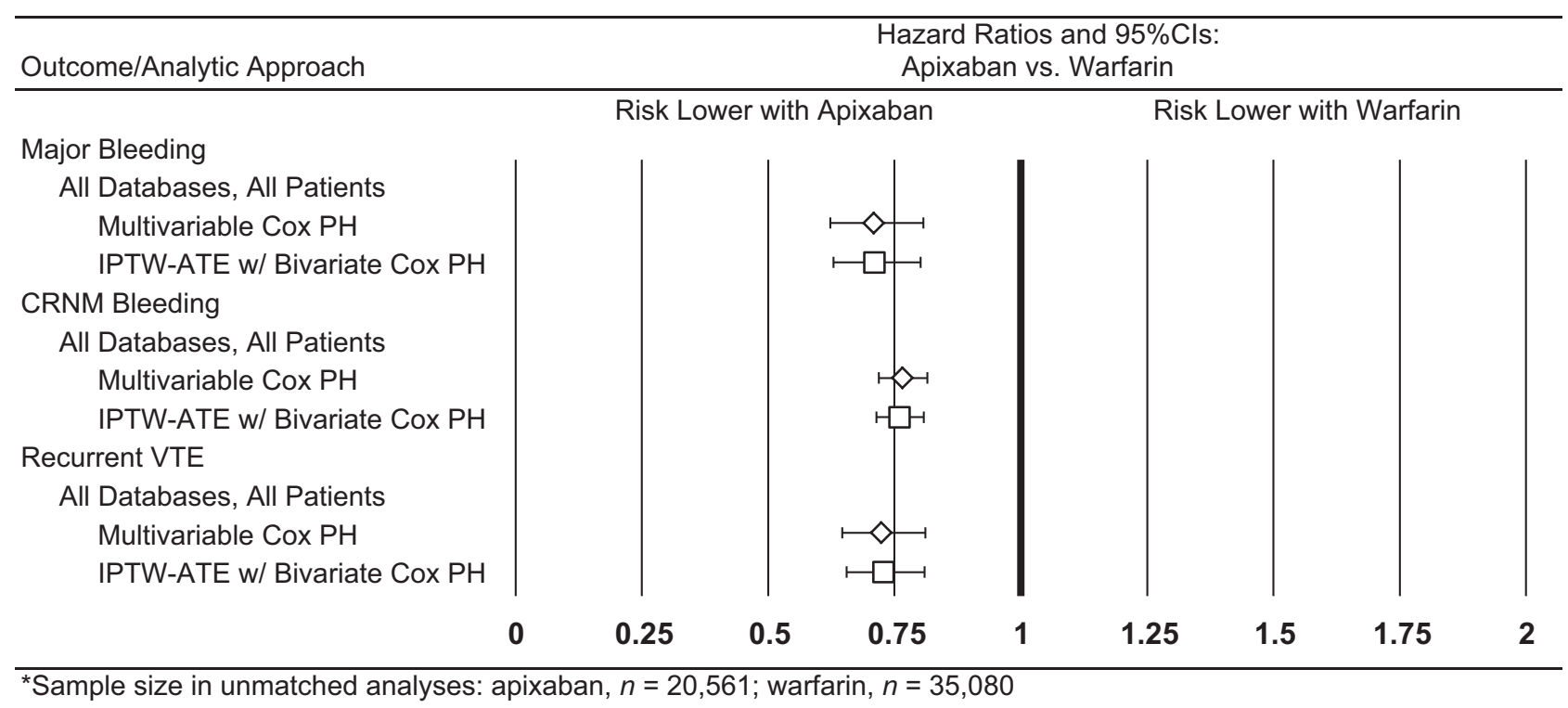

Fig. 4 Hazard ratios for bleeding events and recurrent VTE among patients receiving apixaban and warfarin as outpatient therapy for VTE, based on alternative approaches for confounding adjustment. $\mathrm{Cl}$, confidence interval; CRNM, clinically relevant non-major; IPTW-ATE, inverse probability of treatment weighting-average treatment effect; $\mathrm{PH}$, proportional hazard; VTE, venous thromboembolism.

who receive warfarin with bridging therapy, and to the extent that such differences are unobserved, study results may be biased. Other unmeasurable factors including differences in physician-level, practice-level and plan-level characteristics may also confound the results of both medication exposure (apixaban vs. warfarin) and outcomes (bleeding events, recurrent VTE events). Additionally, data on inpatient drug utilization are not available in the study databases, and thus it is not possible to fully characterize the initial management of VTE requiring inpatient care. While a matchedcohort design has certain advantages (e.g. covariate balance), one of the main disadvantages is the loss of sample/information. Accordingly, sensitivity analyses for each outcome were conducted using data on all patients and the inverse probability of treatment weighting method and multivariable Cox proportional hazards models, respectively. The results from these analyses were similar to those yielded by the matchedcohort design.

Our study used definitions for major bleeding, CRNM bleeding and recurrent VTE that have not been formally validated and cannot be objectively confirmed within the scope of this study, and thus their accuracy is unknown. However, the definition for major bleeding was based in large part on validated claims-based algorithms and the International Society on Thrombosis and Haemostasis' (ISTH) definition that was used in a large clinical trial, and our definition for major bleeding has been employed in previously published studies. ${ }^{33-37}$ Also, our definition for recurrent VTE is similar to ones used in prior evaluations. $^{38-41}$ Because, to the best of our knowledge, there are no recommendations or guidelines for defining CRNM bleeding in real-world studies, we defined CRNM bleeding as events that did not qualify as major bleeding and that did not involve ISTH-defined critical care sites. ${ }^{42}$
Although the health plans contributing claims and enrolment information to the four databases are different, the possibility exists that a patient may be insured by multiple plans at the same time and thus be included in more than one database during the same period. The extent of such overlap is, however, believed to be low. ${ }^{43}$ The possibility also exists that a patient who changed residence or left one health plan to join another could be represented multiple times in the study databases, although over different periods. While information on drug dose and drug supply is available from outpatient pharmacy claims, we cannot determine from such data if dispensed drug was actually taken, when it was taken or how much was taken. Because the study data sources do not include complete mortality information, death was not treated as a competing risk, which may inflate estimated risks of the outcomes of interest. We suspect, however, that the percentage of patients who died during follow-up is low (i.e. < 10\%) based on the observed mortality rate in the AMPLIFY trial, and thus that any such bias does not disproportionately impact in a material fashion one treatment group versus the other. ${ }^{44}$

Because the accuracy of algorithms/variables capturing the presence of acute and chronic conditions is undoubtedly less than perfect, because results from exams (e.g. blood pressure) and laboratories (e.g. serum creatinine, blood glucose, liver enzymes) are not available in the data sources, and because histories are left-truncated, some patients may be misclassified in terms of their comorbidity profile and/or pre-index health care experience. The study population comprised patients with medical and drug benefits from private U.S. health plans, and thus the study population may not reflect patients treated in clinical practice across the United States. Consequently, study results may not be generalizable to those with public health insurance, the uninsured or other segments 
of the U.S. population. Finally, because of the challenges establishing causality in evaluations such as ours, caution should be exercised in interpreting the results of this evaluation and additional research based on data from other realworld settings is needed to validate these findings.

\section{Conclusion}

In this large-scale evaluation of VTE patients receiving outpatient treatment with apixaban or warfarin in U.S. clinical practice, risks of major bleeding, CRNM bleeding and recurrent VTE were found to be significantly lower among patients who received apixaban. These results, which were largely comparable with, and supplement those from, the pivotal clinical trial AMPLIFY, provide the first evidence regarding the effectiveness and safety of apixaban for the treatment of VTE in real-world settings. Additional research based on data from other real-world settings would be helpful in validating these findings.

\section{What is known about this topic?}

- Warfarin has long been standard of care for VTE, despite increased risk of bleeding.

- In recent clinical trial (AMPLIFY), apixaban was noninferior to enoxaparin/warfarin in treatment of VTE and had significantly lower risk of major bleeding.

- Evidence of drug efficacy and safety from clinical trials may not, however, be reflective of their effectiveness and safety in clinical practice.

\section{What does this paper add?}

- This large-scale evaluation provides first evidence on effectiveness and safety of apixaban versus warfarin (plus bridge therapy) in a real-world VTE population.

- Risks of major bleeding, non-major bleeding and recurrent VTE were lower among patients receiving apixaban, consistent with results from AMPLIFY.

\section{Note}

Some aspects of this research were presented at the American College of Cardiology's 67th Annual Scientific Session (March 2018) in Orlando, Florida.

\section{Funding}

Funding for this research was provided to Policy Analysis Inc. (PAI) by Bristol-Myers Squibb (BMS) and Pfizer Inc.

\section{Conflict of Interest}

M.A., A.H. and D.W. are employed by PAI. X.L., G.D.W., M.H., L.V., X.P. and L.B. are employed by, and own stock in, BMS. T.L., X.L. and J.M. are employed by, and own stock in, Pfizer Inc. A.T.C. receives consultancies and honoraria from BMS and Pfizer, Bayer, Daiichi Sankyo, Boehringer Ingelheim and Portola, as well as other companies.

\section{References}

1 Coleman Cl, Baugh C, Crivera C, et al. Healthcare costs associated with rivaroxaban or warfarin use for the treatment of venous thromboembolism. J Med Econ 2017;20(02):200-203

2 Pendergraft T, Liu X, Edelsberg J, et al. Prophylaxis against venous thromboembolism in hospitalized medically ill patients. Circ Cardiovasc Qual Outcomes 2013;6(01):75-82

3 Edelsberg J, Hagiwara M, Taneja C, Oster G. Risk of venous thromboembolism among hospitalized medically ill patients. Am J Health Syst Pharm 2006;63(20, Suppl 6):S16-S22

4 Oster G, Ollendorf DA, Vera-Llonch M, Hagiwara M, Berger A, Edelsberg J. Economic consequences of venous thromboembolism following major orthopedic surgery. Ann Pharmacother 2004;38 (03):377-382

5 Anderson FA Jr, Spencer FA. Risk factors for venous thromboembolism. Circulation 2003;107(23, Suppl 1):I9-I16

6 Cohen AT, Alikhan R, Arcelus JI, et al. Assessment of venous thromboembolism risk and the benefits of thromboprophylaxis in medical patients. Thromb Haemost 2005;94(04):750-759

7 Heit JA, O'Fallon WM, Petterson TM, et al. Relative impact of risk factors for deep vein thrombosis and pulmonary embolism: a population-based study. Arch Intern Med 2002;162(11):1245-1248

8 Spencer FA, Lessard D, Emery C, Reed G, Goldberg RJ. Venous thromboembolism in the outpatient setting. Arch Intern Med 2007;167(14):1471-1475

9 Beckman MG, Hooper WC, Critchley SE, Ortel TL. Venous thromboembolism: a public health concern. Am J Prev Med 2010;38(4, Suppl):S495-S501

10 Kearon C, Akl EA, Ornelas J, et al. Antithrombotic therapy for VTE disease: CHEST guidelines and expert panel report. Chest 2016; 149(02):315-352

11 Eliquis [package insert]. Princeton, NJ: Bristol-Myers Squibb Company; 2017. Available at: http://packageinserts.bms.com/ pi/pi_eliquis.pdf. Accessed August 8, 2017

12 Pradaxa [package insert]. Ridgefield, CT: Boehringer-Ingelheim Pharmaceuticals Inc.; 2017. Available at: http://docs.boehringeringelheim.com/Prescribing\%20Information/PIs/Pradaxa/Pradaxa. pdf. Accessed August 8, 2017

13 Savaysa [package insert]. Tokyo, Japan: Daiichi Sankyo Inc; 2017. Available at: https://www.accessdata.fda.gov/drugsatfda_docs/ label/2015/206316lbl.pdf. Accessed August 8, 2017

14 Xarelto [package insert]. Titusville, NJ: Janssen Pharmaceuticals Inc; 2017. Available at: https://www.xareltohcp.com/shared/product/ xarelto/prescribing-information.pdf. Accessed August 8, 2017

15 Agnelli G, Buller HR, Cohen A, et al; AMPLIFY Investigators. Oral apixaban for the treatment of acute venous thromboembolism. N Engl J Med 2013;369(09):799-808

16 Bauersachs R, Berkowitz SD, Brenner B, et al; EINSTEIN Investigators. Oral rivaroxaban for symptomatic venous thromboembolism. N Engl J Med 2010;363(26):2499-2510

17 Büller HR, Prins MH, Lensin AW, et al; EINSTEIN-PE Investigators. Oral rivaroxaban for the treatment of symptomatic pulmonary embolism. N Engl J Med 2012;366(14):1287-1297

18 Schulman S, Kearon C, Kakkar AK, et al; RE-COVER Study Group. Dabigatran versus warfarin in the treatment of acute venous thromboembolism. N Engl J Med 2009;361(24):2342-2352

19 Büller HR, Décousus H, Grosso MA, et al; Hokusai-VTE Investigators. Edoxaban versus warfarin for the treatment of symptomatic venous thromboembolism. N Engl J Med 2013;369(15):1406-1415

20 Coumadin [package insert]. Princeton, NJ: Bristol-Myers Squibb Company; 2017. Available at: https://packageinserts.bms.com/pi/ pi_coumadin.pdf. Accessed August 8, 2017

21 Kearon C, Akl EA, Comerota AJ, et al. Antithrombotic therapy for VTE disease: antithrombotic therapy and prevention of thrombosis, $9^{\text {th }}$ ed: American College of Chest Physicians Evidence-Based Clinical Practice Guidelines. Chest 2012;141(2, Suppl):e419S-e496S

22 Lee LH. DOACs - advances and limitations in real world. Thromb J 2016;14(Suppl 1):17 
23 Merli GJ, Hollander JE, Lefebvre P, et al. Costs of hospital visits among patients with deep vein thrombosis treated with rivaroxaban and LMWH/warfarin. J Med Econ 2016;19(01):84-90

24 Coleman CI, Turpie AGG, Bunz TJ, Beyer-Westendorf J. Effectiveness and safety of rivaroxaban versus warfarin in patients with provoked venous thromboembolism. J Thromb Thrombolysis 2018;46(03):339-345

25 Coleman CI, Peacock WF, Bunz TJ, Beyer-Westendorf J. Effectiveness and safety of rivaroxaban versus warfarin in patients with unprovoked venous thromboembolism: a propensity-score weighted administrative claims cohort study. Thromb Res 2018;168:31-36

26 Coleman CI, Bunz TJ, Turpie AGG. Effectiveness and safety of rivaroxaban versus warfarin for treatment and prevention of recurrence of venous thromboembolism. Thromb Haemost 2017;117(10):1841-1847

27 Larsen TB, Skjøth F, Kjældgaard JN, Lip GYH, Nielsen PB, Søgaard M. Effectiveness and safety of rivaroxaban and warfarin in patients with unprovoked venous thromboembolism: a propensity-matched nationwide cohort study. Lancet Haematol 2017;4 (05):e237-e244

28 Sindet-Pedersen C, Langtved Pallisgaard J, Staerk L, et al. Comparative safety and effectiveness of rivaroxaban versus VKAs in patients with venous thromboembolism. A Danish nationwide registrybased study. Thromb Haemost 2017;117(06):1182-1191

29 Austin PC. An introduction to propensity score methods for reducing the effects of confounding in observational studies. Multivariate Behav Res 2011;46(03):399-424

30 Normand ST, Landrum MB, Guadagnoli E, et al. Validating recommendations for coronary angiography following acute myocardial infarction in the elderly: a matched analysis using propensity scores. J Clin Epidemiol 2001;54(04):387-398

31 Allison PD. Survival Analysis Using SAS: A Practical Guide. 2nd ed. North Carolina: SAS Institute Inc.; 2010

32 Pencina MJ, D’Agostino RB Sr, D’Agostino RB Jr, Vasan RS. Evaluating the added predictive ability of a new marker: from area under the ROC curve to reclassification and beyond. Stat Med 2008;27 (02):157-172

33 Cunningham A, Stein CM, Chung CP, Daugherty JR, Smalley WE, Ray WA. An automated database case definition for serious bleeding related to oral anticoagulant use. Pharmacoepidemiol Drug Saf 2011;20(06):560-566

34 Thigpen JL, Dillon C, Forster KB, et al. Validity of international classification of disease codes to identify ischemic stroke and intracranial hemorrhage among individuals with associated diagnosis of atrial fibrillation. Circ Cardiovasc Qual Outcomes 2015;8 (01):8-14

35 Granger CB, Alexander JH, McMurray JJV, et al; ARISTOTLE Committees and Investigators. Apixaban versus warfarin in patients with atrial fibrillation. N Engl J Med 2011;365(11): 981-992

36 Li XS, Deitelzweig S, Keshishian A, et al. Effectiveness and safety of apixaban versus warfarin in non-valvular atrial fibrillation patients in "real-world" clinical practice. A propensity-matched analysis of 76,940 patients. Thromb Haemost 2017;117(06): 1072-1082

37 Deitelzweig S, Luo X, Gupta K, et al. Effect of apixaban versus warfarin use on healthcare resource utilization and costs among elderly patients with nonvalvular atrial fibrillation. J Manag Care Spec Pharm 2017;23(11):1191-1201

38 Cai J, Preblick R, Zhang Q, Kwong WJ. Utilization of parenteral anticoagulants and warfarin: impact on the risk of venous thromboembolism recurrence in the outpatient setting. Am Health Drug Benefits 2014;7(08):444-451

39 Laliberté F, Coleman CI, Bookhart B, et al. Weekly risk of venous thromboembolism recurrence in patients receiving oral anticoagulants. Curr Med Res Opin 2014;30(08):1513-1520

40 Lin J, Lingohr-Smith M, Kwong WJ. Incremental health care resource utilization and economic burden of venous thromboembolism recurrence from a U.S. payer perspective. J Manag Care Pharm 2014;20(02):174-186

41 Deitelzweig SB, Lin J, Kreilick C, Hussein M, Battleman D. Warfarin therapy in patients with venous thromboembolism: patterns of use and predictors of clinical outcomes. Adv Ther 2010;27(09):623-633

42 Kaatz S, Ahmad D, Spyropoulos AC, Schulman S; Subcommittee on Control of Anticoagulation. Definition of clinically relevant nonmajor bleeding in studies of anticoagulants in atrial fibrillation and venous thromboembolic disease in non-surgical patients: communication from the SSC of the ISTH. J Thromb Haemost 2015;13(11):2119-2126

43 Broder MS, Neary MP, Chang E, Cherepanov D, Katznelson L. Treatments, complications, and healthcare utilization associated with acromegaly: a study in two large United States databases. Pituitary 2014;17(04):333-341

44 Austin PC, Lee DS, Fine JP. Introduction to the analysis of survival data in the presence of competing risks. Circulation 2016;133 (06):601-609

Erratum: The article was corrected as per Erratum published on January 27, 2020. DOI of the Erratum is 10.1055/s-0039-3400530. 